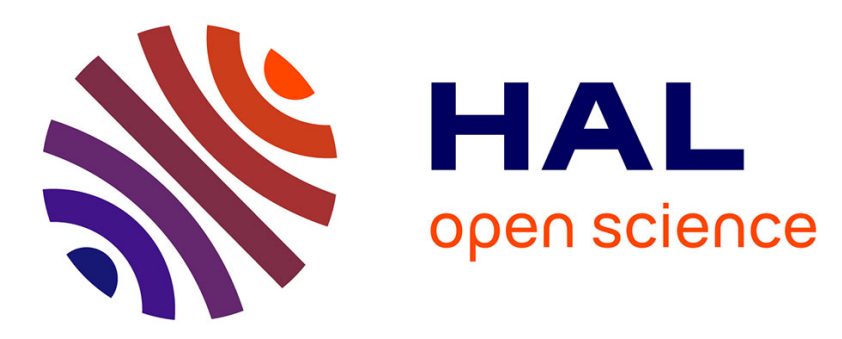

\title{
The crystal structure of the secreted dimeric form of the hemophore HasA reveals a domain swapping with an exchanged heme ligand.
}

Mirjam Czjzek, Sylvie Létoffé, Cécile Wandersman, Muriel Delepierre, Anne Lecroisey, Nadia Izadi-Pruneyre

\section{To cite this version:}

Mirjam Czjzek, Sylvie Létoffé, Cécile Wandersman, Muriel Delepierre, Anne Lecroisey, et al.. The crystal structure of the secreted dimeric form of the hemophore HasA reveals a domain swapping with an exchanged heme ligand.. Journal of Molecular Biology, 2007, 365 (4), pp.1176-86. 10.1016/j.jmb.2006.10.063 . hal-00163418

\section{HAL Id: hal-00163418 https://hal.science/hal-00163418}

Submitted on 30 Aug 2007

HAL is a multi-disciplinary open access archive for the deposit and dissemination of scientific research documents, whether they are published or not. The documents may come from teaching and research institutions in France or abroad, or from public or private research centers.
L'archive ouverte pluridisciplinaire HAL, est destinée au dépôt et à la diffusion de documents scientifiques de niveau recherche, publiés ou non, émanant des établissements d'enseignement et de recherche français ou étrangers, des laboratoires publics ou privés. 


\title{
The crystal structure of the secreted dimeric form of the hemophore HasA
}

\section{reveals a domain swapping with an exchanged heme ligand.}

\author{
Mirjam Czjzek ${ }^{2}$, Sylvie Létoffé ${ }^{3}$, Cécile Wandersman ${ }^{3}$, Muriel Delepierre ${ }^{1}$, Anne \\ Lecroisey $^{1}$ \& Nadia Izadi-Pruneyre ${ }^{1 *}$
}

1:Unité de Résonance Magnétique Nucléaire des Biomolécules CNRS URA 2185 Département de Biologie Structurale et de la Chimie, Institut Pasteur, 75724 Paris Cedex 15 France. 2:Present address: CNRS; Université Pierre et Marie Curie-Paris6, Laboratoire International Associé-Dispersal and Adaptation in Marine Species, Unité Mixte de Recherche 7139, Station Biologique, F-29682 Roscoff Cedex, France. 3: Unité des Membranes Bactériennes, CNRS URA 2172 Département de Microbiologie Fondamemtale et Médicale, Institut Pasteur, 75724 Paris Cedex 15 France.

running title: domain swapped dimeric hemophore

To whom correspondence should be addressed to:

Nadia Izadi-Pruneyre, Unité de Résonance Magnétique Nucléaire des Biomolécules CNRS URA 2185 Département de Biologie Structurale et de la Chimie, Institut Pasteur, 28, rue du Dr. Roux, 75724 Paris Cedex 15 France. tel: 33140613666 fax: 33145688929 e-mail: nizadi@pasteur.fr

The atomic coordinates and structure factor (codes $2 \mathrm{CN} 4$ and $\mathrm{r} 2 \mathrm{cn} 4 \mathrm{sf}$ ) have been deposited in the Protein Data Bank.

Abbreviations used: GdnHCl, guanidine $\mathrm{HCl}$;OD, optical density; PDB, Protein Data Bank; rmsd, root mean square deviation. 


\section{ABSTRACT}

To satisfy their iron needs, several gram-negative bacteria use a heme uptake system involving an extracellular heme-binding protein called hemophore. The function of the hemophore is to acquire free or hemoprotein-bound heme and to transfer it to HasR, its specific outer membrane receptor, by protein-protein interaction. The hemophore HasA secreted by Serratia marcescens, an opportunistic pathogen, was the first to be identified and is now very well characterized. HasA is a monomer that binds one $b$ heme with strong affinity. The heme in HasA is highly exposed to solvent and coordinated by an unusual pair of ligands, a histidine and a tyrosine.

In the present work, we report the identification, the characterization and the X-ray structure of a dimeric form of HasA from Serratia marcesens: DHasA. We show that both monomeric and dimeric forms are secreted in iron deficient conditions by Serratia marcescens. The crystal structure of DHasA reveals that it is a domain swapped dimer. The overall structure of each monomeric subunit of DHasA is very similar to that of HasA but formed by parts coming from the two different polypeptide chains, involving one of the heme ligands. Consequently DHasA binds two heme molecules by residues coming from both polypeptide chains. We show here that, while DHasA can bind two heme molecules, it is not able to deliver them to the receptor HasR. However, DHasA can efficiently transfer its heme to the monomeric form that, in turn, delivers it to HasR. We assume that DHasA can function as a heme reservoir in the hemophore system. 


\section{INTRODUCTION}

Iron is essential for many metabolic pathways. However despite its abundance, it is practically insoluble in the presence of oxygen at a physiological $\mathrm{pH}$ and is thus scarcely available. Heme-containing compounds, which are widespread among vertebrate hosts, are advantageous iron reservoirs for bacterial pathogens. Therefore several Gram-negative bacteria use a heme uptake system involving an extracellular heme-binding protein called hemophore. The function of hemophores is to acquire free or hemoprotein-bound heme and to deliver it to a specific receptor at the cell surface whereby the heme is internalized and then used as iron source.

The hemophore secreted by the opportunistic pathogen Serratia marcescens, HasA, was the first to be identified (1) and is now very well characterized. It is secreted under iron deficiency condition by an ABC- type pathway via a C-terminal secretion signal. This $19 \mathrm{kDa}$ protein binds one $b$ heme molecule with an affinity of $5.310^{10} \mathrm{M}^{-1}(2,3)$. HasA is able to bind free heme or to capture it from other hemoproteins, like hemoglobin. The heme transfer from HasA to its receptor HasR which has a much lower affinity for heme (Ka $\left.510^{6} \mathrm{M}^{-1}\right)$ occurs by protein-protein interaction (4). The next steps of heme internalization, including the heme transfer from the receptor to the periplasm and the recycling and/or ejection of the empty hemophore from the receptor are dependent on the energy given by an inner membrane complex composed of three proteins TonB-ExbB-ExbD (5).

The structure of Has A shows an original $\alpha+\beta$ fold, with an unusual arrangement of the hemebinding site. The $\alpha$ helices are all packed on one side of the molecule and the $\beta$ strands on the other side. The heme is held by two loops at the interface of the $\alpha$ and $\beta$ parts of the molecule and is highly exposed to solvent. The iron atom is ferric and coordinated by the $\mathrm{N} \varepsilon$ of a 
histidine residue on one side (His32) and to the On of a tyrosine residue (Tyr75) on the other (6).

We report here the identification, the characterization and the X-ray structure of a dimeric form of the hemophore present in the culture medium: DHasA. Our results reveal that DHasA is a domain-swapped dimer in which the same structural element is exchanged between the two subunits. It is noteworthy that the exchanged part contains one of the heme ligands, His32.

Domain swapping was first described at the atomic level by Eisenberg and coworkers in 1994 in the crystal structure of diphtheria toxin (7). It is a mechanism by which one protein molecule exchanges a structural "domain" with an identical partner ("domain" is not used in a strict sense). The 'domain' can be as large as an entire tertiary globular domain, or as small as a single element of secondary structure. Currently more than 60 proteins have been identified as domain-swapped protein (8). It has been advanced that the domain swapping plays a role in the regulation of function, in an evolutionary strategy to create protein complexes, or in misfolding and aggregation, but its biological relevance is still unclear (9). In this work, we show that DHasA could function as an efficient heme reservoir in the hemophore system.

\section{MATERIAL AND METHODS}

\section{Expression and purification of the HasA proteins}

ApoHasA proteins, Has A and DHasA, were obtained from the culture supernatant of Escherichia coli strain POP3 (pSYC34PAM) grown at $30^{\circ} \mathrm{C}$ in $\mathrm{M} 9$ glycerol medium and purified as described previously (10). Serratia marcescens SM365 was also grown at $30^{\circ} \mathrm{C}$ in M9 glycerol supplemented with $0.2 \mathrm{mM} 2,2^{2}$-dipyridyl to reduce available iron. 
The holoforms were prepared by adding a freshly prepared hemin solution to the apo-proteins at a ratio of about $120 \%$. The excess of heme was eliminated by gel filtration. All of the samples were in $50 \mathrm{mM}$ sodium phosphate buffer, $\mathrm{pH} 7$ otherwise mentioned.

The HasA concentration was calculated using the previously determined $\varepsilon_{277 \mathrm{~nm}}$ value of 19500 $\mathrm{M}^{-1} \mathrm{~cm}^{-1}(11)$. The same $\varepsilon_{277 \mathrm{~nm}}$ value was used to determine the concentration of DHasA sample in monomeric protein. All absorption measurements were done in a Perkin-Elmer Lambda 2 spectrophotometer using 1 or $0.2 \mathrm{~cm}$ path length cuvettes.

\section{Hemin solution}

Hemin solutions were prepared just before use to prevent heme aggregation. Bovine hemin (Sigma) was dissolved in $150 \mu \mathrm{l}$ of $0.1 \mathrm{~N} \mathrm{NaOH}$, diluted to volume desired by the adequate buffer, and centrifuged. The concentrations of the hemin solutions were measured in $0.1 \mathrm{~N}$ $\mathrm{NaOH}$ using the $\varepsilon_{385(0.1 \mathrm{NaOH})}$ value of $58440 \mathrm{M}^{-1} \mathrm{~cm}^{-1}(12)$

\section{Analytical Ultracentrifugation}

Ultracentrifugation studies were performed in an XLA (Beckmann) analytical ultracentrifuge using $12 \mathrm{~mm}$ path length cells. Purified DHasA samples were $210^{-5} \mathrm{M}$ (monomer concentration) in $20 \mathrm{mM}$ sodium phosphate, $\mathrm{pH}$ 7. The sedimentation/diffusion equilibrium experiments were performed as previously described (2).

\section{Dimer/monomer formation}

The presence of dimeric and monomeric proteins in various culture supernatant was tested by native-PAGE, followed by immunodetection with anti-HasA antibodies and revealed by ECL+ (Amersham) as described previously by Létoffé et al (13). The immunodetection was used to increase the detection threshold of the samples concentration. Purified monomeric and dimeric HasA of known concentrations of $10^{-5}, 10^{-6}$ and $10^{-7} \mathrm{M}$ were used as standard in order to estimate the concentration of secreted protein. 


\section{Heme binding}

Heme binding experiments were performed by UV-visible spectroscopy by following the Soret band at $407 \mathrm{~nm}$. The concentrations of DHasA and of the hemin solution were respectively $1.810^{-6} \mathrm{M}$ (monomer concentration) and $2.2510^{-5} \mathrm{M}$.

\section{Isothermal titration calorimetry (ITC)}

Titrations were performed at $25^{\circ} \mathrm{C}$ using a MicroCal MCS titration calorimeter (MicroCal Inc., Northampton, MA). Proteins and hemin were in $20 \mathrm{mM}$ sodium phosphate, $\mathrm{pH} 7$. Solutions were thoroughly degassed by stirring under vacuum before use. Due to heme adsorption, the calorimeter cell and the microsyringe used for injections were extensively washed after each experiment. For all titrations, the heat of dilution of the ligand was measured by injecting the ligand into the buffer alone or by injecting an excess of ligand into the cell after saturation. The value obtained was subtracted from the heat of reaction to obtain the effective heat of binding. All of the injections were carried out with 3 min intervals.

The theoretical titration curves were fitted to the experimental data using the ORIGIN software (Micorcal). The molar binding stoechiometry and molar binding enthalpy $(\Delta \mathrm{H})$ were determined directly from the fitted data. The affinity constant of DHasA for heme, which is higher than $10^{8} \mathrm{M}$, was too large to be measured by ITC direct titration. Therefore, it was determined by the displacement method (14) using the H32A-H83Q double mutant of the heme binding pocket with $\mathrm{Ka}=2.6 \times 10^{7} \mathrm{M}^{-1}$ and $\Delta \mathrm{H}=-29.2 \mathrm{~kJ} \cdot \mathrm{mol}^{-1}$ as previously described by Deniau et al (3). The Gibbs (binding) free energy $(\Delta \mathrm{G})$ and molar entropy of binding $(\Delta \mathrm{S})$ were calculated using the equation: $\Delta \mathrm{G}=-\mathrm{RT} \ln \mathrm{Ka}=\Delta \mathrm{H}-\mathrm{T} \Delta \mathrm{S}$, where $\mathrm{R}$ is the gaz constant and $\mathrm{T}$ is the temperature in Kelvin.

\section{E.coli growth assays}

Growth stimulation of $\mathrm{C} 600 \Delta \mathrm{hemA}:$ km att $\lambda:$ :hasR by exogenously supplied purified protein was tested as follow. Cells expressing HasR were grown in LB medium supplemented with 
$20 \square \mathrm{g} / \mathrm{ml}$ of delta aminolevulinic acid (LBD) and appropriate antibiotics to an $\mathrm{OD}_{600}$ of 1 and $100 \square 1$ of the resulting culture was mixed with $3 \mathrm{ml}$ of top agar and plated on LBD $0.02 \%$ arabinose. Aliquots of $50 \mu \mathrm{l}$ of serial dilutions of various apo/holo monomer/DHasA were added to wells punched in the solidified agar. Growth around the wells was noted after overnight incubation at $37^{\circ} \mathrm{C}$.

\section{NMR spectroscopy}

NMR samples contained about $1.5 \mathrm{mM}$ HasA or DHasA (monomer concentration) in $50 \mathrm{mM}$ Sodium phosphate buffer, pH 5.6. NMR spectra were recorded on Varian Inova 500 or 600 MHz spectrometers equipped with the VNMR 6.1 software and with Sun Sparc Ultra 2 and

Ultra 5 respectively. ${ }^{1} \mathrm{H}_{-}{ }^{15} \mathrm{~N}$ HSQC experiments (15) were recorded at $30^{\circ} \mathrm{C}$ using $1024 \mathrm{x} 128$ data points and 64 scans. The one-dimensional ${ }^{1} \mathrm{H}$ NMR spectra were acquired at $50^{\circ} \mathrm{C}$ with a spectral width of $200 \mathrm{ppm}, 8 \mathrm{k}$ data points and a recycle delay of $40 \mathrm{~ms}$. Water suppression was achieved by presaturation.

\section{Protein crystallization}

The protein concentration was about $4.0 \mathrm{mg} / \mathrm{ml}$ in $50 \mathrm{mM}$ phosphate buffer at $\mathrm{pH} 7$ and $0.02 \% \mathrm{NaN}_{3}$. Initial crystallization trials were performed with MDL (Molecular Dimensions Limited) and Decode Genetics ("Wizard" 1\&2, Emerald Biostructures), that is, a total of 192 trials in two 96 multi-well crystallization plates from Greiner. These trials were set up using a Cartesian crystallization robot, mixing $200 \mu \mathrm{l}$ of protein with $100 \mu \mathrm{l}$ of reservoir solution.

Crystals were grown by the hanging-drop vapor-diffusion method using 24-welled Linbro plates. Hanging drops on siliconized cover slips were prepared by mixing $2 \mu \mathrm{l}$ of the protein solution with $1 \mu \mathrm{l}$ of the reservoir solution that contained $100 \mathrm{mM}$ Tris buffer at $\mathrm{pH} 8.5,2 \mathrm{M}$ $\mathrm{NaH}_{2} \mathrm{PO}_{4}$ and $2 \mathrm{M} \mathrm{K}_{2} \mathrm{HPO}_{4}$. They were then placed over $0.5 \mathrm{ml}$ of the reservoir solution. All crystallization trials were carried out at $20^{\circ}$ Celsius.

\section{Data collection and processing}


X-ray diffraction data of a DHasA crystal were collected at $100 \mathrm{~K}$ at the European Synchrotron Research Facilities (ESRF, Grenoble, France) beamline ID29 using an ADSC Quantum 4R CCD detector. The crystal was flash-cooled in a liquid-nitrogen stream with 10 $\%(\mathrm{v} / \mathrm{v})$ glycerol as cryoprotectant. The wavelength of the synchrotron X-rays was $0.979 \AA$. The crystal was rotated through $100^{\circ}$ with a $1.0^{\circ}$ oscillation range per frame. The raw data were processed using the program MOSFLM (16) and then merged and scaled using the program SCALA (17).

\section{Structure determination and refinement}

The molecular replacement with the program AMoRe (18) in the resolution range of 9 to 3.1 $\AA$ with the model of monomeric HasA (PDB ID: 1B2V) gave two solutions corresponding to the two monomeric subunits that form the dimer, with an overall correlation coefficient of 0.59 and an R-factor of $37.4 \%$. The refinement was performed using REFMAC5, part of the CCP4-package (17). Water molecules were added using CCP4/warp (19). The stereochemistry of the final structures was evaluated using PROCHECK (20). All refinement statistics are summarized in Table 1.

\section{RESULTS}

\section{Identification of a dimeric form of HasA in solution -DHasA-}

Gel filtration chromatography of the supernatant of POP3 (pSYC34PAM) over-expressing HasA in minimum medium at $30^{\circ} \mathrm{C}$, gives two peaks (Figure 1). Their ratio varies depending on the culture from 10 to $50 \%$. Analysis of the fractions corresponding to these peaks by SDS-PAGE shows that they both contain one protein that migrates like HasA, HasA being the unique protein secreted in $E$. coli overexpressing HasA. The slower migrating peak corresponds to the monomeric form of HasA, previously characterized and studied (2). The 
faster migrating peak has an apparent molecular weight corresponding to twice that of HasA, it will be called DHasA hereafter.

The molecular weight of DHasA was determined by analytical ultracentrifugation with the sedimentation/diffusion equilibrium experiment. The experimental data fit in a satisfactory manner using a one-solute ideal solution model, with a molecule of molecular weight $36900 \pm$ 412 (data not shown). This value is in agreement with that of 38302 for a dimeric form of HasA, the variation remaining within the limits of methods.

\section{DHasA is formed in vivo}

DHasA is secreted by Serratia marcescens in comparable proportions to that observed in E.coli. This observation proves that it is not an artefact of over-expression in E.coli.

DHasA is observed by native-PAGE in the culture supernatant. The formation of DHasA is thus not dependent on purification conditions, especially not on the Ammonium Sulfate precipitation leading to high protein concentration. As observed in Figure 2, DHasA and HasA secreted by $S$. marcescens exist both in cleaved and uncleaved forms. The conditions of cleavage have already been studied for HasA and the study revealed that cleavage is performed by $S$. marcescens major protease, PrtSM, and concerns the twelve C-terminal residues of HasA (21).

The presence of DHasA is not the result of an accumulation of the monomeric form during the culture. Indeed, it is detected during the exponential phase of culture when cell density reaches an $\mathrm{OD}_{600}$ of 1 and when the total Has A protein concentration is estimated to be $10^{-6}$ $\mathrm{M}$ and not only during the stationary phase.

DHasA, like HasA, is secreted in minimum medium in the apoform as shown by the absence of the Soret band at $407 \mathrm{~nm}$, characteristic of the holoform, in the UV-visible spectrum. The holoform can be obtained by adding a solution of hemin to the culture medium. The amount 
of dimeric form obtained is comparable to that obtained when the medium is not supplemented with hemin. The presence (or absence) of heme in the culture medium has thus no influence on DHasA formation.

\section{Structural comparison of DHasA and another dimeric form obtained in vitro}

Three different crystal forms of HasA were obtained under different crystallization conditions (22). The overall structure of HasA in the three crystals is the same. Nevertheless, even though all were obtained from a purified monomeric sample, Has A is in a dimeric form in one of the crystal (PDB ID: 1DK0). Therefore the question arises: are the two dimeric proteins obtained in vitro and in vivo identical? In order to answer this question we have analyzed the structure of DHasA by NMR to determine if it is equivalent to that of $1 \mathrm{DK} 0$.

The ${ }^{15} \mathrm{~N}-{ }^{1} \mathrm{H}$ HSQC spectrum is a fingerprint of the backbone conformation of a protein. Each ${ }^{15} \mathrm{~N}-{ }^{1} \mathrm{H}$ cross-peak reflects amino acid local environment in protein. The comparison of ${ }^{15} \mathrm{~N}$ ${ }^{1} \mathrm{H}$ HSQC spectrum of ApoDHasA with that of ApoHasA for which the complete assignment is known (10) allowed us to obtain structural informations. This spectral comparison was done at two concentrations, namely 0.4 and $1.5 \mathrm{mM}$. At both concentrations, the number of ${ }^{15} \mathrm{~N}-{ }^{1} \mathrm{H}$ amide connectivities present in the ApoDHasA spectrum is comparable to that usually found in the ApoHasA spectrum at the limit of resolution (169 vs 172 respectively). The high similarity between both spectra suggests a high structural homology between the two protein forms.

Most of the residues could be easily assigned by homology. Residues 48-58, 71, 72, 73, 75 and 77 could not be assigned in DHasA spectra. This observation indicates that while the global structure of the protein is conserved, the environment and/or the structure of theses residues are changed indicating that they are involved in dimerisation. Residues 42, 43, 45, 
$67,69,82,83,86,99$ which display slight differences in their chemical shifts as compared to the monomeric form (up to $1 \mathrm{ppm}$ in ${ }^{15} \mathrm{~N}$ and $0.2 \mathrm{ppm}$ in ${ }^{1} \mathrm{H}$ dimension) may be located at the immediate sequential and/or spatial vicinity (backbone and side-chain) of residues at interface. (Figure 3)

The crystal structure of the dimeric form of HasA, 1DK0, could not explain all the differences between the ApoHasA and ApoDHasA spectra. Conversely, residues 88-107 located at the interface of the monomeric subunits of HasA in 1DK0 do not show significant variation of chemical shifts. These observations are indicative of substantial differences in the quaternary structure of DHasA with respect to that of 1DK0. Thus, the dimeric form observed in the crystal structure of $1 \mathrm{DK} 0$ might be an intermediate of aggregation, stabilized in the crystal. Indeed, at high ionic strength (i.e $150 \mathrm{mM} \mathrm{NaCl}$ ) the presence of some aggregated Has A has been detected.

No dissociation of DHasA into monomers was observed by $\mathrm{NMR}$ at $30^{\circ} \mathrm{C}$ at a protein concentration of $1.5 \mathrm{mM}$ during one week and inversely no dimerisation of the monomeric sample was observed at the same or higher concentrations (up to $1.8 \mathrm{mM}$ ). The lack of dissociation and the very high stability of DHasA, generally observed in domain-swapped dimers, suggest that DHasA might be this type of dimer. In order to check this hypothesis, we have thus determined the crystal structure of DHasA and studied properties and activity of this new HasA dimeric form by different methods.

\section{DHasA crystallizes as a domain swapped dimer}

DHasA has successfully been crystallized. The optimized reservoir conditions are $100 \mathrm{mM}$ Tris buffer at $\mathrm{pH} 8.5,2 \mathrm{M} \mathrm{K}_{2} \mathrm{HPO}_{4}$ and $2 \mathrm{M} \mathrm{NaH}_{2} \mathrm{PO}_{4}$. The brownish crystals belonged to space group $\mathrm{P} 2{ }_{1}$, with unit parameters highly resembling those of $1 \mathrm{DK} 0$ (Table 1), and

diffracted to a maximum resolution of $2.3 \AA$. Two molecules are present in the asymmetric 
unit, giving a crystal volume per protein mass $\left(V_{M}\right)$ of $2.17 \AA^{3} \mathrm{Da}^{-1}$ and a solvent content of $43 \%$ by volume (23). The coordinates describing two protein molecules with two heme groups per asymmetric unit were refined to a final $\mathrm{R}$-factor and $\mathrm{R}_{\text {free }}$ of $16.9 \%$ and $22.3 \%$.

The overall fold of each monomeric subunit of DHasA is very similar to that of HasA but formed by parts coming from the two different polypeptide chains present in the asymmetric unit. Each monomeric subunit is composed of seven beta strands packed on one side and four $\alpha$-helices on the other side. But for each subunit residues 2-49 does not belong to the same polypeptide chain than other residues (Figure 4a-c). This crossing over of polypeptide chains to form a multimer assemblage is frequently observed in the so-called domain-swapped dimers. Given the fact that both the monomer and the domain swapped dimer of HasA exist in solution and in stable form, DHasA can thus be considered to be a bona fide example of 3D swapping as defined by Liu \& Eisenberg (7). The backbone superimposition of one subunit of DHasA and the monomeric form of HasA shows a rmsd of 0.93. Significant structural changes occur at the dimer interface of the swapped domain, as compared to the monomeric form. As expected, the highest rmsd value is observed in the hinge loop (43-48) linking the swapped domain to the rest of its subunit. Besides to this, two residues, Asn34 and Gly35 also show high rmsd values, compared to the overall mean value, this being valid for the two subunits composing the dimer as shown in Figure 5a. The overall average B-factor, however shows that these regions do not display particular high B-factors as compared to monomeric HasA (Figure 5b). On the contrary, dimeric form of HasA appears to be less flexible, especially in the region of the heme binding pocket.

The heme binding sites are similar to that of the monomeric form, and are composed of two loops positioned at the interface of the $\alpha$ and $\beta \square$ side that fix the heme in a relatively solvent exposed environment. The swapping makes both heme sites less accessible than that of the monomeric form. The resolution of the data unambiguously revealed that the heme can take 
two orientations, related by a $180^{\circ}$ rotation about the $\alpha, \gamma$-meso axis, as has already been observed in the previously determined HasA structures (22). The heme iron of each monomeric part is coordinated by the On of a tyrosine (Tyr75) and to the $\mathrm{N} \varepsilon$ of a histidine (His32) with the difference that the latter belongs to the swapped region, and the heme molecule of each subunit is actually bound by residues coming from the two polypeptide chains.

\section{Heme environment of DHasA characterization by NMR}

The ${ }^{1} \mathrm{H}$ NMR spectra of HoloDHasA shows the presence of two sets of resonances with relative intensity of 70:30 corresponding to the two possible orientations of the heme observed in the crystal structure (Figure 6). This ratio is well visible in a couple of isolated peaks at 72.5 and $75.7 \mathrm{ppm}$. The same ratio was equally found for the monomeric form as reported by Caillet-Saguy et al (24). The methyl resonances of the two heme molecules are observed between 25 and $50 \mathrm{ppm}$. These chemical shifts are intermediate between those typical for a high spin $\mathrm{S}=5 / 2$ iron (III) heme and those for a low spin $\mathrm{S}=1 / 2$ iron (III) heme. This presumes the existence of a high spin / low spin equilibrium as that observed for the monomeric form (24). The chemical shifts are however slightly different to that of the monomeric form at the same temperature and $\mathrm{pH}$, indicating that the heme iron properties and environment in solution and/or the high spin/low spin species ratio are not exactly the same for the two protein forms.

\section{Activity of DHasA}

The function of HasA is to take up heme and to shuttle it to a specific receptor. To determine the implication of DHasA in the heme acquisition pathway mediated by the hemophore, if any, we have studied the heme binding properties and its delivery to the receptor HasR. 
Heme binding: To study the heme binding, we carried out heme titration experiments by UVvisible spectroscopy and by ITC microcalorimetry as described in our previous works $(2,3,11)$. The titration curve obtained by UV-visible spectroscopy shows that DHasA binds one molecule of heme/monomer (data not shown). The ITC titration data can be fitted without any constraint to a single set of sites model with a stoichiometry of $1 \mathrm{~mol} / \mathrm{mol}$ of monomer and an affinity constant higher than $10^{8} \mathrm{M}^{-1}$ for each site. However, at the minimal concentration that could be used in the calorimetric cell to obtain a reliable heat emission signal, $10^{-5} \mathrm{M}$, the curve was too steep at the equivalence point to determine precisely the affinity constants (Figure 7). Hence, we determined their values by displacement titration using a low affinity mutant the H32A-H83Q: $910^{8} \mathrm{M}^{-1}$ for each site.

From the results of the ITC titration experiments, we could deduce an enthalpy value of $110.5 \pm 2 \mathrm{~kJ} \cdot \mathrm{mol}^{-1}$ and an entropy value of $-59.4 \mathrm{~kJ} \cdot \mathrm{mol}^{-1}$ for the heme binding. The interaction of heme with DHasA is thus strongly enthalpy driven as observed in our previous work (3) for the monomeric form of HasA $\left(-105.4 \pm 3.8 \mathrm{~kJ} \cdot \mathrm{mol}^{-1}\right)$. The decrease in binding entropy in DHasA $\left(\mathrm{T} \Delta \mathrm{S}=-59.4 \mathrm{~kJ} \cdot \mathrm{mol}^{-1}\right)$ compared to HasA $\left(\mathrm{T} \Delta \mathrm{S}=44.3 \mathrm{~kJ} \cdot \mathrm{mol}^{-1}\right)$ is consistent with a decrease of the solvent exposed hydrophobic surface area around the heme from $14 \AA^{2}$ to $10 \AA^{2}$.

The difference of heme iron properties in solution deduced from NMR analysis and the slight difference of some residues position in the loop containing His32, in particular Asn34 and Gly35, linked to the heme propionate groups by hydrogen bonds, can explain the lower affinity of DHasA for the heme molecule. Indeed, besides the residues of the hinge loop, these are the residues that display the highest rmsd values in the superposition of each monomer of DHasA with the crystal structure of monomeric HoloHasA (1B2V). The difference in the positions of Asn34 and Gly35 is only seen in the presence of heme (Figure 5a). 
Heme delivery of DHasA to HasR: Added exogenously, the HoloDHasA does not allow heme acquisition of C600 4hemA::km at $\lambda: \because h a s R$. It has recently been shown that the heme of Has A is transferred to the receptor HasR by protein-protein interaction (4). The inability of HoloDHasA to transfer directly its heme to HasR can be due either to an absence of interaction between these two proteins or to an inability of HoloDHasA to deliver its heme to HasR. The first hypothesis was tested using the solid-phase binding assay. Aliquots of $10^{7}$ CFU of POP3 (pam238-hasR) HasR producing bacteria and of the control strain POP3 (pam238) were spotted onto nitrocellulose membranes and incubated with serial dilutions of apo or HoloDHasA protein. The interaction was probed with a polyclonal antiHasA serum (25). As shown in Figure 8, no binding affinity above background was observed with samples incubated with $10^{-6}, 10^{-7}$ or $10^{-8} \mathrm{M}$ apo or HoloDHasA indicating that the DHasA is unable to bind to the receptor HasR.

Nevertheless in our growth test, HoloDHasA mixed with ApoHasA allows the heme acquisition by bacteria and become thus a source of heme for bacteria. This result indicates that the heme of DHasA is transferred to HasA who delivers it, in turn, to the specific receptor (Table 2).

\section{DISCUSSION}

In this work we have identified a secreted dimeric form of Has A, DHas A. Its structure reveals that DHasA is a domain-swapped dimer. Three-dimensional domain swapping is an event by which a monomer exchanges part of its structure with an identical monomer to form an oligomer, where each subunit has a similar structure to the monomer. While each subunit structure of DHasA compared to that of HasA monomeric form is entirely conserved significant structural changes occur at the dimer interface of the domain-swapping. As expected, the highest rmsd value is observed in the hinge loop (43-48), composed of 
GGFYGG. Although the relation between the hinge loop sequence and swapping phenomenon is not actually demonstrated, the presence of prolines and residues generating, some flexibility have been put forward in facilitating the domain swapping process. Here, the presence of 4 glycine residues in the hinge loop ensures the flexibility needed to perform the swapping. Furthermore, the presence of two hydrophobic residues (FY) constrained at the dimer interface due to both steric hindrance and their hydrophobic character may be a factor that drive the hinge loop to swap or not. The hinge loop sequence is strictly conserved in Erwinia caratovora hemophore (http://www.sanger.ac.uk/Projects/E_caratovora) which is not yet characterized. It is conserved in the form of GXFXXG in all of the other hemophores found in Pseudomonas aeruginosa, P. fluorescence, Yersinia pestis, and Y. enterocolitica. The swapping brings residues Phe45 and Tyr46 close to the 75-85 loop of the same polypeptide chain, creating supplementary hydrophobic interactions among residues of the same chain. As a consequence, Phe78 is in a more hydrophobic environment than that observed in the monomeric form where it is exposed to the solvent.

The swapping in DHasA conserves the overall structure of the heme binding site as well as the ligands of the heme group, although it is noteworthy that one of them, His 32 , is provided by the swapped domain. Nevertheless, some differences are observed in the heme binding pocket of DHasA as compared to that of the monomeric form: i) both heme sites of DHasA are less accessible to solvent because of the swapping and the heme binding pocket appears to be less flexible by the protein-protein interface (lower B-factors in Figure 5b) ii) residues Asn34 and Gly35, which belong to the swapped domain and are hydrogen bonded to the heme propionate groups, show higher rmsd than that of the rest of protein (except for the hinge loop, Figure 5a). These differences can explain the variations of heme chemical shifts observed in solution by NMR and the lower affinity of DHasA for heme compared to that of monomeric form $\left(9 \cdot 10^{8} \mathrm{M}^{-1}\right.$ vs $\left.5,310^{10} \mathrm{M}^{-1}\right)$. 
We have shown here that DHasA exists in the culture supernatant and its presence does not result from over-expression or purification conditions which might expose the protein to destabilizing condition at high concentration. This observation and the very high stability of HasA - Tm of $60^{\circ} \mathrm{C}$ and half unfolding concentration of $\mathrm{GdnHCl}$ of $2.7 \mathrm{M}-(26)$, allow to assume that DHasA folds directly from an unfolded state and by an independent pathway, and not via the initial formation of the monomer. This model has also been proposed by Rousseau and co-workers for suc1, a cell cycle regulatory protein, with two native states, a monomer and a domain swapped dimer $(27,28)$. Assuming that a single polypeptide sequence gives access to the two forms of HasA under the same conditions, both in E.coli and in S.marcescens and this apparently independently of any particular bacterial signal, two questions arise: what are the folding pathways for the monomer and the domain swapped dimer and how are they connected to each other? The fact that HasA of Serratia marcescens is secreted via its C-terminal secretion signal means that the polypeptide needs to be fully synthesized before being secreted. Moreover, the cytoplasmic chaperone SecB has been shown to be essential for HasA secretion in vivo (29). In its absence, folded HasA accumulates in the cytoplasm and is unable to be secreted (30). Together, these data show that the folding of Has A should be achieved during or after secretion. Furthermore, in vitro study of refolding of denatured HasA has demonstrated that Sec B slows down HasA folding. In this study, refolding of denatured HasA has been analyzed by stopped-flow measurements of the fluorescence of its unique tryptophan (26). DHasA had not been characterized before and consequently the mono/oligomeric state of refolded HasA has not been checked in that study. Nevertheless, as the tryptophan, located in the swapped part of DHasA, has exactly the same environment in both monomeric HasA and in DHasA, the formation or the presence of DHasA, if any, could not be detected by the fluorescence signal. The folding/unfolding study of both forms of HasA at different concentrations, while checking the presence and the 
relative amount of each form for example by analysis of the fluorescence anisotropy decay, will provide further information on the folding pathway and contribute to a better understanding of the domain swapping phenomenon.

Currently more than 60 proteins have been identified as domain-swapped oligomers with no apparent structural or functional connection between them. Despite the increasing number of examples, clear evidence for a functional role of domain swapping is lacking (28). Several examples exist for which only the domain swapped version of the protein has been described or only the swapped multimeric form is functional. In some cases, however, both swapped and unswapped forms of the protein have been characterized and domain swapping has been proposed to have a regulatory function. For example, Glyoxalase I from Pseudomonas aeruginosa exists both in an active domain-swapped dimeric state and as a metastable less active monomer (31). In vitro conversion from dimeric to metastable monomeric Glyoxalase I can be triggered upon addition of glutathation. After removal of glutathione, glyoxalase I slowly reverts to a more stable and active domain-swapped form. However, no evidence exists for such a mechanism in vivo. Another interesting finding is that formation of domainswapped oligomers can introduce allosteric regulation of the protein activity already present in the monomer, as shown in bovine seminal Rnase (32). It is intersting to mention the example of Cyanovirin-N for which an identical activity and binding affinity towards gp120 was observed for both the pure monomeric or dimeric protein. Both forms are stable, however the elevated value of the dimerization constant $\left(2.5 \mathrm{mM}\right.$ at $\left.50^{\circ} \mathrm{C}\right)$ suggests that the dimeric form does not exist under physiological conditions (33). In our case, both the monomeric and the domain swapped dimeric forms exist in vivo and are stable and active. The monomeric form of HasA binds one molecule of free or hemoprotein-bound heme and delivers it to a specific outer membrane receptor, HasR, by protein-protein interaction (4). The swapped dimeric form, DHasA, binds two molecules of heme with an affinity constant which remains 
high, even if it is lower than that of the monomeric form. While it is not capable to deliver its hemes directly to the extracellular receptor HasR, since it does not interact with this receptor, it can, however, transfer them to the monomeric form of HasA that, in turn, will interact with HasR to deliver heme. Létoffé et al have shown that two regions of the hemophore are involved in the interaction with HasR, namely the beta strand composed of residues 51 to 60 and the region consisting of residues 95 to 107 (13). These two regions in DHas A become partially inaccessible by dimerization. Furthermore, steric hindrance due to the bigger size of DHasA can be an obstacle for recognition and interaction with HasR. Therefore, we propose that DHasA could serve as a heme reservoir, stocking it and keeping it more accessible for bacteria by the Has system. The efficiency and advantage of DHasA for the Has system would result in its ability to bind two heme molecules with an affinity lower than that of the monomeric form but still high compared to other heme binding proteins in serum, like albumin (the two highest affinities determined for heme-albumine are $7.810^{7} \mathrm{M}^{-1}$ and $2.310^{8}$ $\mathrm{M}^{-1}$ (34). Furthermore, as the $\mathrm{Kd}$ for heme binding of heme receptors from other bacteria is estimated to be in the order of $\mu \mathrm{M}$, the capacity of DHasA to serve as a heme reservoir for the Has system makes the heme acquisition via hemophore efficient and advantageous for Serratia marcescens in the presence of other bacteria sharing the same space.

Acknowledgement

We are indebted to the European synchrotron research facilities (ESRF, Grenoble, France) for beam time allocation for this project and to the staff on beamline ID29 for technical assistance during data collection. We thank Robert Gilli for help with ITC experiments. 
Ref:

1. Létoffé, S., Ghigo, J.M. \& Wandersman. C. (1994) Iron acquisition from heme and hemoglobin by a Serratia marcescens extracellular protein. Proc Natl Acad Sci U S A. 1994 11;91(21):9876-80.

2. Izadi, N., Henry, Y., Haladjian,. J., Goldberg, M.E., Wandersman, C., Delepierre, M., \& Lecroisey, A. (1997) Purification and Characterization of an Extracellular HemeBinding Protein, HasA, Involved in Heme Iron Acquisition. Biochemistry, 36, 70507057.

3. Deniau, C., Gilli, R., Izadi-Pruneyre, N., Létoffé, S., Delepierre ,M., Wandersman, C., Briand, C. \& Lecroisey, A. (2003) Thermodynamics of heme binding to the HasA(SM) hemophore: effect of mutations at three key residues for heme uptake. Biochemistry. 16;42(36):10627-33.

4. Izadi-Pruneyre, N., Huche, F., Lukat-Rodgers, G.S., Lecroisey, A., Gilli, R., Rodgers, K.R., Wandersman, C. \& Delepelaire, P. (2006) The heme transfer from the soluble HasA hemophore to its membrane-bound receptor HasR is driven by protein-protein interaction from a high to a lower affinity binding site. $J$ Biol Chem. Sep $1 ; 281(35): 25541-50$.

5. Létoffé, S., Delepelaire, P. \& Wandersman, C. (2004) Free and hemophore-bound heme acquisitions through the outer membrane receptor HasR have different requirements for the TonB-ExbB-ExbD complex. J Bacteriol. 186(13):4067-74.

6. Arnoux, P., Haser, R., Izadi, N., Lecroisey, A., Delepierre, M., Wandersman, C. \& Czjzek, M. (1999) The crystal structure of HasA, a hemophore secreted by Serratia marcescens. Nat Struct Biol. Jun;6(6):516-20.

7. Bennett, M.J., Choe, S. \& Eisenberg, D. (1994) Refined structure of monomeric diphteria toxin at 2.3 A resolution. Protein Sci. 3: 1464-1475. 
8. Yang, S., Cho, S.S., Levy, Y., Levine, H., Wolynes, P.G. \& Onuchic, J.N. (2004) Domain swapping is a consequence of minimal frustration. Proc Natl Acad Sci U S A. 101 (38) 13786-13791.

9. Liu, Y. \& Eisenberg, D. (2002) 3D domain swapping: as domains continue to swap. Protein Sci. 11(6):1285-99. Review.

10. Izadi-Pruneyre, N., Wolff, N., Castagné, C., Czisch, M., Wandersman, C., Delepierre, M. \& Lecroisey A. (1999) Backbone NMR assignment and secondary structure of the 19 kDa hemophore HasA” J. Biomol. NMR, 14 (2), 193-194.

11. Létoffé, S., Deniau, C., Wolff, N., Dassa, E., Delepelaire, P., Lecroisey, A. \& Wandersman, C. (2001) Haemophore-mediated bacterial haem transport: evidence for a common or overlapping site for haem-free and haem-loaded haemophore on its specific outer membrane receptor. Mol Microbiol. 41(2):439-50.

12. Dawson, R.M.C, Elliott, D.C. Elliott, W.H. \& Jones, K.M. (1986) in Data for Biochemical Research, pp 230-231, Oxford University Press, Oxford, England.

13. Létoffé, S., Debarbieux, L., Izadi, N., Delepelaire, P. \& Wandersman, C. (2003) Ligand delivery by haem carrier proteins: the binding of Serratia marcescens haemophore to its outer membrane receptor is mediated by two distinct peptide regions. Mol Microbiol. 50(1):77-88.

14. Sigurskjold BW. (2000) Exact analysis of competition ligand binding by displacement isothermal titration calorimetry. Anal Biochem.15; 277(2):260-6.

15. Kay, L.E., Keifer, E.P. \& Saarinen, T (1992). Pure absorption gradient enhanced heteronuclear single quantum correlation with improved sensitivity. J Am Chem Soc. 114: 10663-10665.

16. Leslie, A.G.W. (1990). in 'Crystallographic computing', Oxford University Press, pp $50-61$. 
17. Collaborative Computational Project, Number 4, CCP4. (1994) Acta Crystallogr. D $50,760-763$.

18. Navaza, J. (1994). AmoRe: an automated package for molecular replacement. Acta Crystallogr A 50, 157-163.

19. Perrakis, A., Sixma, T. K., Wilson, K. S., and Lamzin, V. S. (1997). wARP: improvement and extension of crystallographic phases by weighted averaging of multiple-refined dummy atomic models. Acta Crystallogr. D 53, 448-455.

20. Laskowski, R.A., MacArthur, M.W., Moss, D.S. \& Thornton, J.M. (1993) PROCHECK: a program to check the stereochemical quality of protein structures. $J$ Appl Cryst 26: 283-291.

21. Izadi-Pruneyre, N., Wolff, N., Redeker, V., Wandersman, C., Delepierre, M. \& Lecroisey, A. (1999) NMR studies of the C-terminal secretion signal of the haembinding protein, HasA. Eur J Biochem. 261(2):562-8.

22. Arnoux, P., Haser, R., Izadi-Pruneyre, N., Lecroisey, A. \& Czjzek, M. (2000) Functional aspects of the heme bound hemophore HasA by structural analysis of various crystal forms. Proteins. 1;41(2):202-10.

23. Matthews, B. W. (1968). Solvent content of protein crystals. J. Mol. Biol. 33, 491-497.

24. Caillet-Saguy, C., Delepierre, M., Lecroisey, A., Bertini, I., Piccioli, M. \& Turano, P. (2006) Direct-detected 13C NMR to investigate the iron(III) hemophore HasA. J Am Chem Soc. 11;128(1):150-8.

25. Létoffé, S., Nato, F., Goldberg, ME. \& Wandersman, C. (1999) Interactions of HasA, a bacterial haemophore, with haemoglobin and with its outer membrane receptor HasR. Mol Microbiol. 33(3):546-55. 
26. Wolff, N., Sapriel, G., Bodenreider, C., Chaffotte, A. \& Delepelaire P. (2003) Antifolding activity of the $\operatorname{SecB}$ chaperone is essential for secretion of HasA, a quickly folding ABC pathway substrate. J Biol Chem. 3;278(40):38247-53.

27. Rousseau, F., Schymkowitz, J.W. Wilkinson, H.R. \& Itzhaki, L.S. (2001) Proc Natl Acad Sci U S A. 8,98(10):5596-601. Three-dimensional domain swapping in p13suc1 occurs in the unfolded state and is controlled by conserved proline residues.

28. Rousseau, F., Schymkowitz, J.W. \& Itzhaki, L.S. (2003) The unfolding story of threedimensional domain swapping. Review. Structure 11(3):243-5.

29. Delepelaire, P. \& Wandersman, C. (1998) EMBO J. The SecB chaperone is involved in the secretion of the Serratia marcescens HasA protein through an ABC transporter. Feb16;17(4):936-44.

30. Debarbieux, L. \& Wandersman, C. (2001) Folded HasA inhibits its own secretion through its ABC exporter. EMBO J. 3;20(17):4657-63.

31. Saint-Jean, A.P., Phillips, K.R., Creighton, D.J \& Stone, M.J. (1998) Biochemistry. 21;37(29):10345-53. Active monomeric and dimeric forms of Pseudomonas putida Glyoxalase I : evidence for 3D domain swapping.

32. Vitagliano, L., Adinolfi, S., Sica, F., Merlino, A., Zagari, A., \& Mazzarella, L. (1999). A potential allosteric subsite generated by domain swapping in bovine seminal ribonuclease. J Mol Biol. 29, 293(3):569-77.

33. Barrientos, L.G., Gronenborn, A.M. (2005) The highly specific carbohydrate-binding protein cyanovirin-N: structure, anti-HIV/Ebola activity and possibilities for therapy. Mini Rev Med Chem.5(1):21-31

34. Gattoni, M., Boffi, A., Sarti, P. \& Chiancone, E. (1996) Stability of the heme-globin linkage in alphabeta dimers and isolated chains of human hemoglobin. A study of the 
heme transfer reaction from the immobilized proteins to albumin. J Biol Chem. 26;271(17):10130-6. 


\section{Figure Legends:}

Figure 1:Gel filtration on a HiPrep 16/60 Sephacryl S-100 High resolution equilibrated with $50 \mathrm{mM}$ sodium phosphate buffer, $\mathrm{pH}$ 7. The flow rate was $30 \mathrm{~mL} / \mathrm{h}$. The volume of each fraction was $1.5 \mathrm{~mL}$.

Figure 2:DHasA is directly observed in the culture supernatant of S.marcescens in iron limiting condition. Native-PAGE followed by immunodetection with anti-HasA antibody (1/2000) was used for its observation. Lane 1: monomeric purified HasA from E. coli as standard $\left(\right.$ Has $\left.\mathrm{A}_{\mathrm{Ec}}\right)$; lane 2: Purified DHasA from E. coli as standard (DHasA $\mathrm{Ac}_{\mathrm{Ec}}$ ); Lanes 3 and 4: 0,2 and $0,6 \mathrm{OD}_{600}$ equivalent from the supernatant of $\mathrm{SM} 365$ grown in minimal medium supplemented with 0,4 mM 2.2'-dipyridyl (iron-limiting conditions). Has $\mathrm{A}_{\mathrm{Sm}}$ and $\mathrm{DHas}_{\mathrm{Sm}}$ are secreted by $S$. marcescens.

Figure 3: Superimposition of $600 \mathrm{MHz}{ }^{1} \mathrm{H}_{-}{ }^{15} \mathrm{~N}$ HSQC spectra of HasA (in black) and DHasA (in red) $\left(1.5 \mathrm{mM}\right.$ in $20 \mathrm{mM}$ sodium phosphate buffer, $\left.\mathrm{pH} 5.6,30^{\circ} \mathrm{C}\right)$. Both spectra were obtained using the same acquisition and processing parameters.

Figure 4: (A) Ribbon representation of the backbone of domain swapped dimeric DHasA (PDB ID: 2CN4). The two chains A and B are colored in red and blue, heme is in yellow. (B) One half DHasA composed of two polypeptide chains (blue/red). (C) Final refined electron density map in the region of the domain swapping, contoured at a $1 \sigma$ level. Chain A is colored in red and chain B is colored in yellow. 
Figure 5: (A): R.m.s. deviation calculated by superimposing the backbone atoms of the monomeric form (1B2V) and the individual subunits of DHasA. The highest r.m.s deviation values observed for residues marked with a star are discussed in the text. The continuous and broken lines are related to molecule A and B of DHasA respectively. (B) Average B factor of all atoms as a function of residue number for DHasA molecule A (solid line), molecule B (grey squares) and compared to those of monomeric Has A (dashed line). The residues in the hinge region (43-48) of the swapping domain show comparable B-factors in both forms of protein.

Figure 6: ${ }^{1} \mathrm{H}$ NMR spectra of HoloHasA (A) and HoloDHasA (B) recorded at $500 \mathrm{MHz}$ and at $50^{\circ} \mathrm{C}$ in $20 \mathrm{mM}$ sodium phosphate buffer, $\mathrm{pH}$ 5.6.

Figure 7: Isothermal Titration Calorimetry (ITC) of the interaction of DHasA with hemin in $50 \mathrm{mM}$ sodium phosphate buffer $\mathrm{pH} 7$ at $25^{\circ} \mathrm{C}$. The heat signal is shown (upper) together with the binding isotherm derived from this signal (lower). Protein concentration was $5.610^{-6} \mathrm{M}$ (monomer concentration). Injections of $10 \mu \mathrm{l}$ of a hemin solution at $5.910^{-5} \mathrm{M}$ were performed with an interval of $3 \mathrm{~min}$. The ITC titration data were fitted without any constraint to a single set (or class) of sites model with a stoichiometry of $1.024 \pm 0.005(\mathrm{~mol} / \mathrm{mol}$ of monomer), an affinity constant higher than $>10^{8} \mathrm{M}^{-1}$ and a $\Delta \mathrm{H}$ observed of $-108.8( \pm 1.3)$ $\mathrm{kJ} . \mathrm{mol}^{-1}$ (or $-26.1 \mathrm{Kcal}_{\mathrm{mol}}{ }^{-1} \pm 0.3$ ) for each site.

Figure 8: Solid phase binding assays. Each sample was tested in triplicate at concentration indicated on the figure. The dot blots were probed with polyclonal anti-HasA serum at a 1/2000 dilution, revealed by ECL + (1/30000) and scanned with storm imager. Same results were obtained with holoproteins. 